\title{
Intrathecal baclofen in the treatment of adult spasticity
}

\author{
JosePh C. Hsieh, M.D., M.B.A., M.P.H., AND RichaRd D. Penn, M.D. \\ Section of Neurosurgery, University of Chicago, Illinois
}

\begin{abstract}
$\checkmark$ Medical management of adult spasticity, a condition of increased muscle tone and deep tendon reflexes, is often challenging and complex. Oral medications such as baclofen often have unacceptable supraspinal side effects at effective doses. Intrathecal baclofen delivered by an implanted catheter and pump system provides good relief of spasticity while overcoming these limitations. In this paper the authors survey the use of oral and intrathecal baclofen therapy, detail the surgical process, and explain the risks and benefits of the procedure.
\end{abstract}

KEY WORDS • spasticity • intrathecal baclofen • implantable pump

$\mathrm{S}$ PASTICITY is simply defined as a state of increasing muscle tone with movement and associated exaggerated deep tendon reflexes. ${ }^{17}$ The simplicity of its description, however, belies the seriousness of the condition. The excessive involuntary motor activity associated with spasticity can result from lesions of the CNS. It may manifest in forms including, but not limited to, a Babinski response; exaggerated stretch, cutaneous, or autonomic reflexes; and abnormal postures (dystonia). ${ }^{39}$ In short, spasticity remains a multifaceted entity, and because of this, multiple treatment modalities have been attempted.

Therapies for spasticity have run the gamut of medical and surgical innovation. Conservative options include the use of traditional oral medications and rehabilitative therapies. Oral pharmacology for spasticity has been confined to medications that limit peripheral cholinergic activity at the neuromuscular junction (for example, botulinum toxin), that inhibit the release of calcium from the sarcoplasmic reticulum (for example, dantrolene sodium), or modulate CNS activity (for example, baclofen, diazepam, and clonidine). ${ }^{21,24}$

When medical therapy has failed, surgery has at times resulted in success. The best-known surgery, modern selective dorsal rhizotomy, can be traced back to operations credited to Otfried Foerster ${ }^{12}$ in 1913. Selective dorsal rhizotomy involves the surgical sectioning of select dorsal nerve roots that exhibit abnormal activation on EMG studies. The positive results include increases in voluntary mobility and reductions in rigidity. Nevertheless, risks include symptom recurrence and loss of sphincter control and sensation. ${ }^{10,11}$ Other surgical lesioning options such as ventral rhizotomy, cordectomy, or midline myelotomy have all met with limited success but with even more significant morbidity. ${ }^{32}$

In recent years, a synergistic approach including med-

Abbreviations used in this paper: $\mathrm{BBB}=$ blood-brain barrier; $\mathrm{CNS}=$ central nervous system; $\mathrm{CP}=$ cerebral palsy; $\mathrm{CSF}=$ cerebrospinal fluid; EMG = electromyography; GABA $=\gamma$-aminobutyric acid; ITB = intrathecal baclofen. ical and surgical options has been found to be particularly effective. Perhaps the most promising and rapidly adopted surgical therapy for spasticity has been an implanted pump catheter for the delivery of ITB. In this paper we will detail an introduction to intrathecal and oral baclofen, the surgical process, and risks and benefits associated with the procedure.

\section{Baclofen}

Baclofen, whose chemical name is 4-amino-3-(4chlorophenyl)-butanoic acid (Lioresal), was approved in 1977 by the Food and Drug Administration for treatment of spasticity (Fig. 1). Historically, baclofen had been designed as an antiepileptic drug in the 1920s. Although its effect on epilepsy was disappointing, investigators found that in certain patients spasticity decreased. Indeed, baclofen is currently the most effective and widely used drug for treatment of spinal cord or cerebral spasticity.

Baclofen is an analog to GABA that is specific to the $\mathrm{GABA}_{\mathrm{B}}$ (G protein-coupled) receptor. ${ }^{3}$ Oral GABA, a hydrophilic agent, is an ineffective antispasmodic drug because it lacks penetration through the BBB and is rapidly degraded by neural tissue. Baclofen (molecular weight 213.67) is slightly more lipid-soluble and stable due to its chlorophenyl moiety, and thus it crosses the BBB in significantly high concentrations without being taken up by cells.

Baclofen has been found to bind to presynaptic $\mathrm{GABA}_{\mathrm{B}}$ receptors within the brainstem, dorsal horn of the spinal cord, and other CNS sites. ${ }^{5,13,20,22,25}$ There is evidence to suggest that baclofen may work at a spinal level. Specifically, baclofen administered to isolated spinal cord preparations inhibits both monosynaptic and polysynaptic reflexes. ${ }^{40}$ Presynaptic effects are believed to be secondary to decreases in calcium influx during an action potential, leading to reduced neurotransmitter release. The challenge with baclofen has been to develop a method of delivery that will minimize supraspinal side effects while maintaining efficacy. 

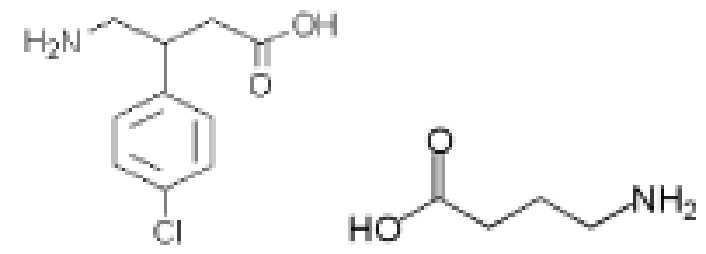

Fig. 1. Schematics showing the molecular structure of baclofen (left) and GABA (right).

\section{Oral Baclofen}

Baclofen is most frequently delivered through an oral route. Oral baclofen has been shown to be an effective antispasmodic agent by Duncan, et al. ${ }^{7}$ Baclofen appears to work best on spasticity caused by multiple sclerosis and spinal cord injury, although some efficacy is also seen in patients with head trauma or CP. The usual dosages for spasticity range from 60 to $100 \mathrm{mg} /$ day. Gradual increases in doses may be necessary as the disease process evolves, but can also result in tolerance.

Severe spasticity is rarely controlled by oral baclofen, although some relief of symptoms is possible. ${ }^{32}$ As might be suspected from its central $\mathrm{GABA}_{\mathrm{B}}$ inhibitory action, the risks associated with oral baclofen include central side effects of drowsiness and mental confusion. Other risks include dependence and withdrawal symptoms, seizures, psychic symptoms, and hyperthermia. Luckily, withdrawal symptoms are usually mitigated by the reintroduction of baclofen.

In open-label studies of oral baclofen, the drug improved spasticity in 70 to $87 \%$ of patients, with additional improvement in spasms in 75 to $96 \%$ of patients. ${ }^{6}$ Doubleblind, cross-over, placebo-controlled trials demonstrate statistically significant improvements in spasticity with administration of oral baclofen. The $\alpha_{2}$-adrenergic agonist tizanidine has been the medication most often compared with oral baclofen and has been found to be similarly efficacious but with a reduced frequency of weakness.

\section{Intrathecal Baclofen}

The supratentorial CNS side effect profile of baclofen provides an upper limit to oral baclofen dosing. The suppression of abnormal spinal segment reflex activity in severe spasticity can require oral dosing, which results in clinically hazardous levels of sedation caused by cerebral effects. The most successful solution to this dilemma has been direct delivery of baclofen into the lumbar subarachnoid space with an intrathecal pump.

Intrathecal baclofen represents a significant advancement in drug delivery that bypasses several limitations of oral baclofen. The technology has its roots in successful CNS delivery of intrathecal opiods, resulting in prolonged analgesia with minimal systemic side effects. ${ }^{38}$ Intrathecal delivery of baclofen overcomes the obstacles of the BBB and theoretically results in greater therapeutic efficacy concentrated at a spinal site of action. Because baclofen is only slightly lipid soluble, it remains within the CSF, with a relatively long half-life of 90 minutes. ${ }^{26}$ Furthermore, slow rostral perfusion of baclofen along the subarachnoid space creates a relatively high concentration of the drug in the spine compared with the brain. Proof of this principle was demonstrated during initial trials in which a single 50$\mu \mathrm{g}$ bolus of baclofen introduced into the lumbar region reduced severe rigidity in patients with spinal cord injury to normal tone for more than 8 hours, without cerebral side effects. ${ }^{33}$

An implanted drug pump provides continuous infusion within the lumbar subarachnoid space and thus makes it possible to sustain an antispasmodic baseline. Furthermore, dosing can easily be titrated to attain the desired tone simply by adjusting the rate of infusion. In one double-blind cross-over study, patients with severe spinal spasticity refractory to oral therapy were evaluated in an "on-baclofen" and "off-baclofen" state, and they demonstrated significant reduction in both Ashworth and spasm frequency scale scores. ${ }^{34}$ Efficacy was maintained over the course of 2 years.

The only approved pump and catheter system available for ITB therapy is produced by Medtronic, Inc., Minneapolis, Minnesota. The infusion system currently in use consists of an intrathecal catheter, pump, and external computer programmer. The pump is based on systems that are also used for intrathecal/epidural delivery of morphine for chronic pain, intravascular delivery of chemotherapeutic drugs such as methotrexate, doxorubicin, or cisplatin in patients with cancer, and intravenous infusion of clindamycin for osteomyelitis. The largest pump available, the SynchroMed II (Medtronic, Inc.), is $87.5 \mathrm{~mm}$ in diameter, $26 \mathrm{~mm}$ thick, has a capacity of $40 \mathrm{ml}$, and weighs $215 \mathrm{~g}$ when full. The pump is powered by a permanent lithium battery that cannot be recharged in situ; consequently, the pump must be surgically explanted and a new one inserted every 4 to 6 years with normal use. Normal refill intervals are usually between 2 and 3 months.

\section{Surgical Process}

Although it is usually well tolerated, implantation of an intrathecal catheter and baclofen pump represents an invasive surgical procedure that must be fully evaluated before it is undertaken. Care must be taken in patient selection, surgical implantation, and postoperative management.

\section{Patient Selection}

Adequate patient assessment for ITB pump implantation is necessary to determine if surgical intervention is a viable option. Screening requires a complete physical examination and neurological assessment, including a thorough history of spasticity and prior treatment regimens. Common ratings of spasticity include the following: 1 ) the Ashworth Scale (scores 1-5) for assessment of muscle tone in lower extremities (including hip abduction, hip flexion, knee flexion, and ankle dorsiflexion) and upper extremities (including shoulder abduction, elbow extension, elbow flexion, and wrist extension); 2) the Penn Spasm Frequency Scale (scores 0-4) for quantification of spontaneous sustained flexor and extensor muscle spasms per hour; and 3) the Reflex Scale (scores 0-5) for categorization of deep tendon reflexes at the biceps, patella, and Achilles tendon.

One set of reasonable criteria for inclusion in an ITB trial includes the following items: 1) severe chronic spastic hypertonia in the lower extremities lasting at least 6 
months, with Ashworth scores of at least 3 in an affected extremity, or a Penn Spasm frequency score of at least 2 during screening; 2) failure to respond to maximum recommended doses of antispasmodic medications, including baclofen and possibly diazepam, clonidine, tizanidine, or dantrolene sodium.

As part of surgical screening, patients should demonstrate a positive clinical response to an ITB injection bolus. Screening involves a $50-\mu \mathrm{g}$ bolus injection of baclofen diluted to $50 \mu \mathrm{g} / \mathrm{ml}$ into the intrathecal space by barbotage over a period of not less than 1 minute. The patient is then observed over a period of 4 to 8 hours. A positive response consists of a significant decrease in muscle tone, frequency of spasms, or severity of spasms. Patients with suboptimal responses may be rescreened after a rest period of 24 hours with $75 \mu \mathrm{g}$ baclofen $(100 \mu \mathrm{g}$ if necessary). Higher intrathecal doses are rarely if ever indicated. Some patients have clear, classic signs and symptoms of spasticity that have always been known to respond. In such cases, the ITB screening test may be omitted at the discretion of the physician.

\section{Pump Implantation}

The implantation of an intrathecal baclofen pump and catheter is a relatively straightforward surgical procedure. The continuous-infusion pump and spinal catheter systems are usually inserted after induction of general anesthesia with the patient placed in the lateral position after receiving appropriate perioperative antibiotic drugs. Intrathecal catheter placement is best performed with the aid of fluoroscopic guidance by using a percutaneous technique, in which a 14- or 15-gauge Tuohy needle is introduced into an appropriate lower lumbar interspace to avoid the conus. We have found that an insertion slightly lateral to the spinous process with oblique advancement in the intrathecal space reduces the risk of positional catheter occlusion or tearing by normal spine flexion and extension.

The catheter is then threaded into the upper lumbar or lower thoracic region. Care should be taken not to torque or pivot the Tuohy needle while threading the catheter, because this may lead to shearing of the catheter into the intrathecal space. Similarly, the catheter should never be withdrawn without simultaneous needle withdrawal, because this also predisposes the catheter to shearing. The catheter tip should be advanced adequately to prevent migration of the device from the intrathecal space. Conversely, overly aggressive advancement predisposes to a higher cervical and brainstem dose of baclofen in the setting of bulk rostral CSF flow. Although higher catheter tip placement may be indicated in upper-extremity spasticity, we have found T9-L1 to be an appropriate catheter tip level in the majority of cases. Intrathecal catheters may be secured to lumbar fascia without occluding the catheter itself by a simple purse-string suture in the tissue around the Tuohy needle prior to removal. Basic care should be taken with catheter tunneling.

Placement of the continuous-infusion pump itself is a matter of choice. The standard placement has been within the lower abdominal wall in a suprafascial pocket no more than 1 inch from the surface of the skin for ease of refilling. Consideration must be made for patient comfort because the pump may abut either the lower ribs or iliac crest in a mobile patient capable of assuming a seated position. A small lipectomy may be necessary in obese patients to simplify routine postoperative pump filling. Postoperative care may include supine positioning for several hours postimplantation to reduce the risk of postoperative headache and CSF leakage.

\section{Optimal Programming}

Optimal pump programming depends on the patient, and dosing ultimately depends on the severity and location of symptoms, catheter position, and the patient's sensitivity to baclofen. According to our experience, the initial continuous-administration daily dose is usually approximately twice the effective trial bolus dose. Further adjustments may be made after evaluation of the response profile of the patient. Optimal dosing may usually be attained with constant-flow pump titration in two to six adjustments over a period of 1 to 6 months. The most critical time is the initial stabilization period, with dose adjustments made at subsequent refills. Recent 1-year mean daily doses reported in the literature have ranged from 175 to $477 \mu \mathrm{g} /$ day. $^{2,37}$

It is necessary to remember that dosing depends not only on spasticity relief but also on overall patient satisfaction. Therefore, baclofen dosing is rarely as simple as optimizing performance based on spasticity scales. Indeed, Van Schaeybroeck, et al., ${ }^{36}$ reported a blind dose-reduction test conducted in patients with $\mathrm{CP}$ that demonstrated subjective functional deterioration in five but increased Ashworth Scale scores in only two patients, suggesting that spasticity is only a single component of the benefit to patients from ITB. Several authors have suggested that subjective functional evaluations are influenced dramatically by baclofenrelated reduction in spasticity-related pain. ${ }^{1}$ Other complicating factors in optimal dosing are the complexity of the clinical syndromes (especially in supraspinal spasticity conditions like (CP) and fluctuating external influences on the patient's condition.

\section{Risks}

Several risks are involved with placement of baclofen pumps. Stempien and Tsai ${ }^{35}$ published perhaps the single most comprehensive study of ITB complications in a survey of 40 centers with a total experience of 1002 test doses and 936 pump placements. Common test-dose complications were nausea/vomiting $(2.6 \%)$ and sedation $(2.2 \%)$. Pump complications included CSF collection (3.3\%), constipation (2.9\%), and headache (2.4\%). Common long-term complications were catheter kink or migration (4\%) and infection (1.2\%).

Even with safe surgical techniques, some common complications of baclofen pump placement still occur. Excessive swelling may be a sign of a developing seroma, sometimes requiring percutaneous drainage. A CSF leak may be detected by a spongy swelling around the incision site, sometimes characterized by a ripple effect when tapped. Standard measures to reduce local CSF pressure at the level of the defect, such as having the patient lie flat without a pillow, are often sufficient. Other measures include using a blood patch and/or abdominal binder.

In the product manual, Medtronic warns of specific risks 
related to the pump itself. For instance, heating of local tissue in circumstances such as short-wave diathermy within $30 \mathrm{~cm}$ of the pump or catheter has been shown to elevate temperatures and cause baclofen overinfusion. Sources of electromagnetic interference like magnetic resonance imaging will temporarily stop the pump motor's rotor due to the magnetic field of the imager, leading to brief disruption of intrathecal therapy. Furthermore, increases in environmental pressure have been found to lead to slower infusion rates. Therefore, a patient must be warned before participating in activities such as scuba diving. Conversely, lower pressures will lead to higher infusion rates. Patients participating in extended high-altitude activities or stays may therefore require readjustment.

A special note should be made about the risks of infection. Normal hallmarks of infection such as warmth or redness around the incision site should be observed and monitored closely. More ominous signs, such as purulent drainage, should be dealt with on an emergency basis, because severe infections may lead to meningitis. Pump removal and intravenously administered antibiotic drugs appropriate for the infecting bacteria are then indicated. In general, infection rates for baclofen pumps implanted to deliver the drug in an effort to treat spasticity appear to range from 0.7 to $1.7 \% .^{30,35}$ Meningitis in patients with implanted pumps ranges from 0 to $0.7 \% .^{28}$ Infections of the pump rather than the reservoir or catheter appear to be most prevalent in the literature. Reported infections appear to involve the perioperative period after placement of the pump. The most common offending bacteria are Staphylococcus aureus or S. epidermidis. Repeated percutaneous refills appear to carry little risk of infection. One theory suggests that the host-derived albumin coating of the pump pocket reduces the risk of colonization. ${ }^{37}$ There are also reports of colonization of implanted pump reservoirs by bacteria and fungus without clinical infection. ${ }^{31}$

Intrathecal use of baclofen does not completely eradicate the central risks. The most frequent drug-related side effects of ITB include drowsiness, dizziness, constipation, and muscular hypotonia. ${ }^{4} \mathrm{~A}$ bolus dose that is too high can result in rostral progression of hypotonia, followed by brainstem toxicity as the baclofen is carried up the neuraxis by rostral bulk CSF flow. Brainstem effects include respiratory depression, hypotension, bradycardia, and coma. Continuous infusion generally has a safer profile than bolus infusion. Malfunctions in prototype pumps have also been noted in the literature, resulting in milligram levels of baclofen release and leading to coma, although newer pump models have not shown this defect. ${ }^{27}$

In cases of severe overdose, the pump should be stopped immediately. Simple airway, breathing, and circulation considerations must be addressed, with mechanical ventilation, intravenous fluids, and vasopressors as supportive measures. Pump interrogation is preferred if a programmer is available. In cases in which pump malfunction is the cause, the device should be turned off if necessary. A 20-ml syringe and 22-gauge Huber needle may be used for access to the fill port for removal of medication. In cases in which the acute overdose is caught within a few hours of the event, a withdrawal of 30 to $40 \mathrm{ml}$ of CSF through the catheter access port may remove the most concentrated baclofen in the CSF (high-volume lumbar puncture has a similar effect). Cases identified beyond this time window are not amenable to CSF removal because the drug has already undergone diffusion and flow up the spinal canal. Although baclofen has no direct antagonist, drowsiness and respiratory depression may be reversed with 1 to $2 \mathrm{mg}$ of intravenous physostigmine over 5 to 10 minutes.

Baclofen withdrawal is a serious risk if there is an abrupt decrease in drug infusion. Causes include catheter failure (for example, obstruction, fracture, or dislodging of the intrathecal catheter), pump malfunction, or low pump reserves. Symptoms include pruritis without a rash, diaphoresis, hyperthermia, hypotension, changes in mental status, and aggravation of spasticity. Continued withdrawal may result in rhabdomyolysis or multiple organ failure, or it may mimic autonomic dysreflexia, sepsis, malignant hyperthermia, or neuroleptic-malignant syndrome. Management involves adequate evaluation of recent painful stimuli, listening for pump alarms, pump interrogation, abdominal and/or spine x-ray films, or indium infusion studies to evaluate for catheter fracture and baclofen extravasation. Once a diagnosis is made, oral baclofen may be administered if tolerated. In severe withdrawal, intrathecal baclofen administration through lumbar puncture may be necessary. Additional intravenous benzodiazepine therapy titrated according to effect may prove useful.

Although they are uncommon, the risk of baclofen-related seizures deserves a special discussion. Interestingly, both overdose and withdrawal of baclofen appear to induce seizures, especially in cases of supraspinal spasticity. Rates of seizure activity in patients receiving boluses of intrathecal baclofen are as high as $10.3 \%$ in cases of spasticity with supraspinal origin..$^{15}$ Overdose-related seizures may be caused by rostral baclofen bulk flow to supraspinal tissue. Withdrawal seizures may occur as baclofen is eliminated from lipid stores and brain tissue. ${ }^{9}$ The seemingly paradoxical anti- and proconvulsant effects of baclofen may depend on the location of $\mathrm{GABA}_{\mathrm{B}}$-related inhibition (for example, on excitatory or inhibitory neurons in traumatized neuronal tissue). In each case, sudden drug level changes seem to have been most critical in the seizure-related activity.

Tolerance is another risk that deserves special attention. Several factors may require progressively higher doses of ITB. Tolerance necessitates increasing doses of medication, but it can be limited on occasion by a drug holiday. Some centers report success with intrathecal morphine during this holiday. Others have used intrathecal fentanyl, although it has not been approved by the Food and Drug Administration for this indication. ${ }^{8}$ Progressive disease, decubitus ulcers, and infections (urinary or systemic) may also require readjustment of dosing.

\section{Benefits}

There is little doubt that ITB therapy fills a critical role in the treatment of spasticity. The primary benefit of ITB is the relief of severe spasms and spasticity. Overall, patients report increased independence, mobility, and ability to perform self-care. Some patients report that they have a more consistent sleep pattern at night; others report renewed ability to have sexual intercourse. Urinary function improves in some as detrusor hyperreflexia and bladder contractions are curtailed. Muscle pain and fatigue accompanying spasm is minimized with ITB, partly because of 
the reduction in spasms, and possibly also because baclofen acts as an antagonist to substance $\mathrm{P}$ in suppressing central pain. ${ }^{14}$ Ultimately, the dose level of ITB is set in a fashion to relieve spasticity while preserving voluntary movement and limiting side effects. Oral baclofen may be weaned over several weeks to prevent withdrawal symptoms of delirium, hallucinations, or seizures.

Reflexes have been found to be particularly improved. Latash, et al., ${ }^{18}$ observed mono- and polysynaptic reflexes and voluntary movements in the lower extremities in patients with chronic refractory spasticity treated with a single bolus of ITB. Responses on EMG studies to joint movements, H-reflexes, ankle clonus, and defensive reactions in the lower extremities were significantly reduced within 30 to 45 minutes and almost completely suppressed by 2 hours. There was also improvement in selective voluntary activation of leg muscles in those with residual motor control. Because ITB exerts an almost purely spinal effect with little or no supraspinal effect due to its concentration gradient, this evidence suggests that supraspinal central motor commands may be somewhat intact despite long-term spasticity in some patients.

Intrathecal baclofen reduces nocturnal disturbance caused by spasticity. Little, et al., ${ }^{19}$ reported diurnal variation in different types of spasticity, with flexor spasms occurring most frequently at night in $50 \%$ of patients. Indeed, spasticity interfered with sleep in $82 \%$ of patients with incomplete lesions and in 50\% of patients with complete lesions. Baclofen infusion improves sleep by reducing these spasms. The activity per hour of the tibialis anterior muscles was reduced during sleep, according to results of EMG studies performed after baclofen infusion, and this lower activity led to reduced sleep disturbance. ${ }^{16}$

Baclofen appears to work in patients suffering from spasticity of either spinal or cerebral origin. For instance, Ordia, et al., ${ }^{29}$ studied 59 patients suffering from severe spasticity of spinal cord origin that was refractory to oral baclofen (for example, spinal cord injury, multiple sclerosis, familial spastic paraparesis, spinal cord tumor, cervical spondylotic myelopathy, transverse myelitis, and amyotrophic lateral sclerosis). In this series, the mean Ashworth rigidity score significantly decreased, from 4.3 to 1.4 $(\mathrm{p}<0.0005)$, and the spasm frequency score decreased from 3.6 to $0.5(\mathrm{p}<0.0005)$.

Baclofen also appears to work in patients suffering from cerebral spasticity. Meythaler, et al.,22 studied 17 patients with stroke who had chronic spasticity lasting longer than 6 months, in whom the Ashworth lower-extremity scores were reduced an average of 2 points during screening. In this series, the mean Ashworth score in the lower extremities after pump implantation had declined at 1 year from 3.7 to 1.8 ( $\mathrm{p}<0.0001$ ), the spasm score from 1.2 to 0.6 $(p=0.43)$, and the reflex score from 2.4 to $1(p<0.0001)$. The mean Ashworth score in the upper extremity declined from 3.2 to $1.8(\mathrm{p}<0.0001)$, the spasm score from 0.7 to $0.3(p=0.87)$, and the reflex score from 2.4 to $1.5(p=0.33)$.

In another study, Meythaler, et al., ${ }^{23}$ evaluated 13 patients with $\mathrm{CP}$ who had intractable spastic hypertonia and quadriparesis refractory to oral medications who dropped an average of 2 points on their Ashworth lower-extremity scores during screening. In this series, the mean Ashworth score in lower extremities after pump implantation had declined at 1 year from 3.4 to 1.5 ( $p<0.0001)$, the spasm score from 1.4 to $0.6(\mathrm{p}=0.1024)$, and the reflex score from 2.5 to $0.7(\mathrm{p}<0.0001)$. The mean upper-extremity Ashworth score decreased from 3 to 1.7 ( $p<0.0001$ ), the spasm score from 1.2 to $0.2(\mathrm{p}=0.0135)$, and the reflex score from 2.3 to 0.5 ( $\mathrm{p}<0.0001)$.

Intrathecal baclofen appears to be cost-effective at an institutional level. Ordia, et al., ${ }^{29}$ found a reduction in the length of hospital stays but no change in overall use of outpatient resources during the 1st year after pump implantation. Patients reduced their mean hospital stays of 7.9 days/year preimplantation to 5.7 days/year in the $1 \mathrm{st}$ year postimplantation, with a cost savings of $\$ 6750$ per patient (excluding the cost of pump implantation). Assuming that reduction in hospital days continues beyond the 1st year, the cost of implanting the system is paid back in less than 2.5 years on average.

\section{Conclusions}

Intrathecal baclofen delivered through an implanted catheter and pump system represents a significant advancement in the management and treatment of adult spasticity. The procedure is generally safe and well tolerated. Most important, intrathecal delivery maximizes spinal effect while minimizing cerebral side effects. Indeed, ITB offers perhaps the best option in many patients with severe spasticity refractory to medical management.

\section{References}

1. Albright AL: Spastic cerebral palsy. Approaches to drug treatment. CNS Drugs 4:17-27, 1995

2. Armstrong RW, Steinbok P, Cochrane DD, Kube SD, Fife SE, Farrell K: Intrathecally administered baclofen for treatment of children with spasticity of cerebral origin. J Neurosurg 87: 409-414, 1997

3. Bowery NG, Hill DR, Hudson AL: $\left[{ }^{3} \mathrm{H}\right](-)$ Baclofen: an improved ligand for $\mathrm{GABA}_{B}$ sites. Neuropharmacology 24:207-210, 1985

4. Bushman W, Steers WD, Meythaler JM: Voiding dysfunction in patients with spastic paraplegia: urodynamic evaluation and response to continuous intrathecal baclofen. Neurourol Urodyn 12:163-170, 1993

5. Coffey RJ, Cahill D, Steers W, Park TS, Ordia J, Meythaler JM, et al: Intrathecal baclofen for intractable spasticity of spinal origin results of a long-term multicenter study. J Neurosurg 78: 226-232, 1993

6. Dario A, Tomei G: A benefit-risk assessment of baclofen in severe spinal spasticity. Drug Saf 27:799-818, 2004

7. Duncan GW, Shahani BT, Young RR: An evaluation of baclofen treatment for certain symptoms in patients with spinal cord lesions: a double-blind cross-over study. Neurology 26: 441-446, 1976

8. Erickson DL, Blacklock JB, Michaelson M, Sperling KB, Lo JN: Control of spasticity by implantable continuous flow morphine pump. Neurosurgery 16:215-217, 1985

9. Faigle JW, Keberle H: The chemistry and kinetics of Lioresal. Postgrad Med J 48 (Suppl 5):9-13, 1972

10. Fasano VA, Barolat-Romana G, Zeme S, Squazzi A: Electrophysiological assessment of spinal circuits in spasticity by direct root stimulation. Neurosurgery 4:146-151, 1979

11. Fasano VA, Broggi G, Barolat-Romana G, Squazzi A: Surgical treatment of spasticity in cerebral palsy. Child Brain 4:289-305, 1978

12. Foerster O: On the indications and results of the excision of posterior spinal nerve roots in men. Surg Gynecol Obstet 16: 463-474, 1913 
13. Ford B, Greene P, Louis ED, Petzinger G, Bressman SB, Goodman R, et al: Use of intrathecal baclofen in the treatment of patients with dystonia. Arch Neurol 53:1241-1246, 1996

14. Herman RM, D'Luzansky SC, Ippolito R: Intrathecal baclofen suppresses central pain in patients with spinal lesions. A pilot study. Clin J Pain 8:338-345, 1992

15. Kofler M, Kronenberg MF, Rifici C, Saltuari L, Bauer G: Epileptic seizures associated with intrathecal baclofen application. Neurology 44:25-27, 1994

16. Kravitz HM, Corcos DM, Hansen G, Penn RD, Cartwright RD, Gianino J: Intrathecal baclofen: effects on nocturnal leg spasticity. Am J Phys Med Rehabil 71:48-52, 1992

17. Lance JW: Symposium synopsis, in Feldman RG, Young RR, Koella WP (eds): Spasticity: Disordered Motor Control. Chicago: Year Book, 1980, pp 485-494

18. Latash ML, Penn RD, Corcos DM, Gottlieb GL: Short-term effects of intrathecal baclofen in spasticity. Exp Neurol 103: 165-172, 1989

19. Little JW, Micklesen P, Umlauf R, Brittell C: Lower extremity manifestations of spasticity in chronic spinal cord injury. Am J Phys Med Rehabil 68:32-36, 1989

20. Meythaler JM: Pharmacology update: intrathecal baclofen for spastic hypertonia in brain injury. J Head Trauma Rehabil 12: 87-90, 1997

21. Meythaler JM: Use of intrathecally delivered medications for spasticity and dystonia in acquired brain injury, in Yaksh TL (ed): Spinal Drug Delivery. New York: Elsevier, 1999, pp 513-554

22. Meythaler JM, DeVivo MJ, Hadley M: Prospective study on the use of bolus intrathecal baclofen for spastic hypertonia due to acquired brain injury. Arch Phys Med Rehabil 77:461-466, 1996

23. Meythaler JM, Guin-Renfroe S, Law C, Grabb P, Hadley MN: Continuously infused intrathecal baclofen over 12 months for spastic hypertonia in adolescents and adults with cerebral palsy. Arch Phys Med Rehabil 82:155-161, 2001

24. Meythaler JM, McCary A, Hadley MN: Intrathecal baclofen for spastic hypertonia in adult brain injury. Perspect Neurosurg 7: 99-107, 1996

25. Meythaler JM, Steers WD, Tuel SM, Cross LL, Haworth CS: Continuous intrathecal baclofen in spinal cord spasticity: a prospective study. Am J Phys Med Rehab 71:321-327, 1992

26. Müller H, Zierski J, Dralle D, Krauss D, Mutschler E: Pharmacokinetics of intrathecal baclofen, in Müller H, Zierski J, Penn RD (eds): Local-Spinal Therapy of Spasticity. Berlin: Springer-Verlag, 1988, pp 223-226
27. Muller-Schwefe G, Penn RD: Physostigmine in the treatment of intrathecal baclofen overdose. J Neurosurg 71:273-275, 1989

28. Ochs G, Naumann C, Dimitrijevic M, Sindou M: Intrathecal baclofen therapy for spinal origin spasticity: spinal cord injury, spinal cord disease, and multiple sclerosis. Neuromodulation 2:108-119, 1999

29. Ordia JI, Fischer E, Adamski E, Spatz EL: Chronic intrathecal delivery of baclofen by a programmable pump for the treatment of severe spasticity. J Neurosurg 85:452-457, 1996

30. Ordia JI, Fischer E, Adamski E, Chagnon KG, Spatz EL: Continuous intrathecal baclofen infusion by a programmable pump in 131 consecutive patients with severe spasticity of spinal origin. Neuromodulation 5:16-24, 2002

31. Penn RD: Intrathecal baclofen for spasticity of spinal origin: seven years of experience. J Neurosurg 77:236-240, 1992

32. Penn RD: Medical and surgical treatment of spasticity. Neurosurg Clin N Am 3:719-727, 1990

33. Penn RD, Kroin JS: Intrathecal baclofen alleviates spinal cord spasticity. Lancet 1:1078, 1984 (Letter)

34. Penn RD, Savoy SM, Corcos D, Latash M, Gottlieb G, Parke B, et al: Intrathecal baclofen for severe spinal spasticity: a doubleblind crossover. N Engl J Med 320:1517-1521, 1989

35. Stempien L, Tsai T: Intrathecal baclofen pump use for spasticity: a clinical survey. Am J Med Rehabil 79:536-541, 2000

36. Van Schaeybroeck P, Nuttin B, Lagae L, Schrijvers E, Borghgraef C, Feys P: Intrathecal baclofen for intractable cerebral spasticity: a prospective placebo-controlled, double-blind study. Neurosurgery 46:603-612, 2000

37. Van Wijngaerden E, Peetermans WE, Vandersmissen J, Van Lierde S, Bobbaers H, Van Eldere J: Foreign body infection: a new rat model for prophylaxis and treatment. J Antimicrob Chemother 44:669-674, 1999

38. Wang JK, Nauss LA, Thomas JE: Pain relief by intrathecally applied morphine in man. Anesthesiology 50:149-151, 1979

39. Young RR: Treatment of spastic paresis. New Eng J Med 320: $1553-1555,1989$

40. Zieglgansberger W: Dorsal horn neuropharmacology: baclofen and morphine. Ann N Y Acad Sci 531:150-156, 1988

Manuscript received June 16, 2006.

Accepted in final form July 12, 2006.

Address reprint requests to: Richard D. Penn, M.D, Section of Neurosurgery, University of Chicago, 5841 South Maryland Avenue, Chicago, Illinois 60637. email: penn@surgery.bsd.uchicago.edu. 